\title{
Intenção materna de amamentar: revisão sistemática
}

\author{
Maternal intention to breastfeed: a systematic review
}

\author{
Tatiana de Oliveira Vieira ${ }^{1}$ \\ Camilla da Cruz Martins ${ }^{1}$ \\ Géssica Silva Santana ${ }^{1}$ \\ Graciete Oliveira Vieira ${ }^{1}$ \\ Luciana Rodrigues Silva ${ }^{2}$
}

${ }^{1}$ Departamento de Saúde, Universidade Estadual de Feira de Santana. Av. Transnordestina s/n, Novo Horizonte. 44036-331 Feira de Santana BA Brasil.

tatianaovieira@gmail.com ${ }^{2}$ Serviço de

Gastroenterologia e

Hepatologia Pediátricas,

Faculdade de Medicina,

Universidade Federal da

Bahia. Salvador BA Brasil.
Abstract This study seeks to identify the factors associated with maternal intention to breastfeed (MIB) in pregnant women. A systematic review of articles in the Medical Literature Analysis and Retrieval System Online (PubMed/Medline) and the Latin American and Caribbean Literature in Health Sciences (Lilacs) databases was conducted. Two independent reviewers performed the search, using the "Medical Subject Headings" and "Key words in Science and Health" vocabulary, referring to: breastfeeding, intention, and pregnant women. Research articles in English, Portuguese and Spanish that analyzed MIB as an outcome were selected. The studies were classified by methodological quality, using a scale that considered internal and external validity criteria, with a maximum score of 30 points. Nine articles were selected, with a methodological quality varying from 12 to 21 points. The studies assessed 68 variables; 35 of which were associated with MIB. The characteristics associated with MIB found in two or more studies were: primiparity, higher level of schooling and older maternal age, prior experience with breastfeeding, not smoking, and living with a partner. Knowledge of the characteristics associated with MIB represents a fundamental step in the planning of actions that seek to increase the duration of breastfeeding.

Key words Systematic review, Intention, Breastfeeding
Resumo O presente estudo tem como objetivo identificar os fatores associados à intenção materna para amamentar (IMA) em gestantes. Foi realizada revisão sistemática de estudos de bases de dados Medical Literature Analysis and Retrieval System Online (PubMed/Medline) e Literatura Latino-Americana e do Caribe em Ciências da Saúde (Lilacs). Dois revisores independentes buscaram artigos, com os "Medical Subject Headings" e os "Descritores em Ciência e Saúde", referentes: ao aleitamento materno, à intenção e às gestantes. Foram incluidas pesquisas que apresentavam IMA como desfecho, em inglês, português e espanhol; e classificadas, quanto à qualidade metodológica, de acordo com critérios de validade interna e externa de uma pesquisa, com pontuação máxima de 30 pontos. Selecionou-se nove artigos, com qualificação metodológica entre 12 e 21 pontos, que investigaram 68 variáveis; 35 dessas se associaram à IMA. As características associadas à IMA em gestantes, relatadas em dois ou mais estudos, foram: primeira gestação, maior escolaridade e idade materna, experiência prévia com a amamentação, ausência do hábito de fumar $e$ residir com o companheiro. O conhecimento das características associadas à IMA representa um passo fundamental no delineamento de ações que visem maior duração do aleitamento materno.

Palavras-chave Revisão sistemática, Intenção, Aleitamento materno 


\section{Introdução}

Nas últimas décadas, a intenção materna de amamentar (IMA) tem sido destacada como uma característica associada ao aleitamento na primeira hora de vida e à maior duração do aleitamento materno $(\mathrm{AM})^{1}$. Muitos estudos foram publicados nessa linha de pensamento; no entanto, poucas são as pesquisas conduzidas com o propósito de saber os fatores que caracterizam a IMA, comportamento que antecede ao ato de amamentar.

Acredita-se que a IMA pode ser definida por diferentes fatores, como: ter experiência prévia com a amamentação $0^{2-6}$, morar com o companheiro $^{3-7}$, primiparidade ${ }^{3,6-11}$, ser mãe não fuman$\mathrm{te}^{3,7,11}$, maior idade materna ${ }^{2,3,8}$, escolaridade materna mais elevada ${ }^{2,7,8,11}$, conhecimento materno dos benefícios do $\mathrm{AM}^{12}$ e apoio paterno ${ }^{13,14}$.

Além desses fatores, também é estudada a relação entre a IMA e a Teoria do Comportamento Planejado ${ }^{15,16}$, que fundamenta a intenção comportamental, em três pilares: (1) atitude comportamental, que é a postura individual em relação a um determinado comportamento; (2) norma subjetiva, que é a percepção pessoal quanto às expectativas e pressões sociais em executar um comportamento específico; e, (3) o controle conhecido do comportamento, que reflete a autoconfiança e a capacidade do sujeito em executar comportamento específico.

Assim, a intenção de realizar um determinado comportamento e a sua efetivação estão relacionadas. No que diz respeito à prática da amamentação, a IMA é o resultado de um comportamento construído de modo complexo e progressivo desde o período gestacional, que nem sempre obedece a um total controle voluntário da nutriz, pois inclui a participação da criança e as pressões do ambiente.

O agregamento das evidências científicas com síntese dos resultados das pesquisas publicadas, no que diz respeito aos fatores que definem a ausência do desejo materno de amamentar, manifestada durante a gestação, pode ajudar os profissionais de saúde a compreenderem este comportamento, bem como a intervirem nas características modificáveis, com o intuito de encorajar a prática de amamentar. Neste sentido, o presente artigo teve como objetivo identificar os fatores associados à intenção materna para amamentar (IMA) em gestantes, mediante revisão sistemática da literatura.

\section{Métodos}

Estudo de revisão sistemática da literatura sobre IMA, a busca de dados incluiu artigos publicados e indexados nas bases de dados Medical Literature Analysis and Retrieval System Online (Pub$\mathrm{Med} / \mathrm{Medline}$ ), através da plataforma na National Center for Biotechnology Information $(\mathrm{NCBI})^{17}$, e na Literatura Latino-Americana e do Caribe em Ciências da Saúde (Lilacs), através da Biblioteca Virtual em Saúde (BVS) ${ }^{18}$.

As buscas e as análises dos artigos foram realizadas por dois revisores independentes, durante os meses de setembro, outubro e novembro de 2013, sendo utilizados os termos breastfeeding, intention, pregnancy, gestational mothers, pregnant woman segundo os Medical Subject Headings (MeSH), e "aleitamento materno, leite humano, intenção, gestantes, gravidez", conforme os Descritores em Ciências da Saúde (DeCS). Não houve restrições quanto ao ano de publicação. A seleção dos artigos foi feita de acordo com os critérios de inclusão: artigos encontrados nas bases de dados com a ocorrência simultânea dos termos de busca, mediante uso de indicadores boleanos "and" e "and not", pesquisas originais que apresentavam a IMA como desfecho principal ou secundário, em qualquer tempo da gestação; e, publicados nos idiomas inglês, português e espanhol. Foram aceitos estudos de intervenção e também os observacionais, qualitativos ou quantitativos, de caráter prospectivo ou retrospectivo. Os artigos de revisão sistemática ou narrativa foram excluídos.

As informações relativas aos artigos selecionados foram consolidadas por dois revisores independentes, com auxílio de um formulário estruturado, no qual foram registrados os nomes dos autores, revista, ano de publicação, título do artigo, local e período de realização, desenho de estudo, número de sujeitos, população do estudo, análise de dados, variáveis estatisticamente associadas ou não à IMA. Nos casos de discordância entre os revisores, no que diz respeito à tabulação dos estudos, esses foram avaliados por um terceiro.

Os artigos que atenderam às condições de inclusão foram qualificados metodologicamente, com pontuação segundo critérios adaptados de outros estudos eleitos como referência ${ }^{19,20}$, com valorização dos requisitos básicos para garantir a validade interna e externa da pesquisa, além de considerar a estrutura e a apresentação do artigo. Os critérios de qualificação e seus respectivos escores encontram-se na Tabela 1. Para cada item foi considerado "escore zero" quando a informa- 
Tabela 1. Critérios de qualificação e escores utilizados na revisão sistemática.

\begin{tabular}{|c|c|}
\hline Critérios de qualidade & Escore \\
\hline Escala de validade interna (tipo de estudo) & 0 a 5 \\
\hline Intervenção randomizado e controlado & 5 \\
\hline Coorte & 4 \\
\hline Caso-controle & 3 \\
\hline Corte transversal & 2 \\
\hline Relato de caso ou série de casos & 1 \\
\hline Não especificado no artigo & 0 \\
\hline Resumo estruturado* & 0 a 1 \\
\hline Introdução com embasamento e justificativa* & 0 a 1 \\
\hline Método de recrutamento da população & 0 a 3 \\
\hline Nacional & 3 \\
\hline Residentes locais (cidade/ bairro) & 2 \\
\hline Usuários de clínicas e serviços & 1 \\
\hline Não especificado no artigo & $\mathbf{0}$ \\
\hline Seleção da amostra & 0 a 6 \\
\hline Censo & 6 \\
\hline Aleatória simples & 5 \\
\hline Sistemática & 4 \\
\hline Estratificada & 3 \\
\hline Por conglomerados & 2 \\
\hline Conveniência & 1 \\
\hline Não especificado no artigo & 0 \\
\hline Instrumentos de coleta de dados & 0 a 3 \\
\hline Validado e padronizado & 3 \\
\hline Validado & 2 \\
\hline Padronizado & 1 \\
\hline Não especificado no artigo & 0 \\
\hline Taxa de não resposta informada ${ }^{*}$ & 0 a 1 \\
\hline Treinamento dos entrevistadores ${ }^{*}$ & 0 a 1 \\
\hline Método de medida de resultados definidos ${ }^{*}$ & 0 a 1 \\
\hline Realizado análise estatística* & 0 a 1 \\
\hline Hipótese do estudo e vieses considerados ${ }^{*}$ & 0 a 1 \\
\hline Resultados interpretados segundo evidências ${ }^{*}$ & 0 a 1 \\
\hline Escala de generalização dos resultados & 0 a 5 \\
\hline Qualquer lugar do mundo & 5 \\
\hline Continentes ou subcontinente semelhante & 4 \\
\hline Mesmo país & 3 \\
\hline Mesma região geográfica & 2 \\
\hline População específica & 1 \\
\hline Não especificado no artigo & 0 \\
\hline Pontuação máxima & 30 \\
\hline
\end{tabular}

"Pontuação igual a zero quando a informação não estava especificada no texto ou quando não atendia ao critério. Adaptado de: Parry et al..$^{19} \mathrm{e}$ Taylor BJ et al. ${ }^{20}$.

ção não estava especificada no texto, ou quando não atendia aos respectivos critérios de classificação de qualidade. $\mathrm{Na}$ avaliação individual de cada um dos artigos previamente selecionados a pontuação máxima possível foi de 30 pontos.

A seleção das variáveis que integraram os grupos seguiu os padrões da epidemiologia des- critiva, no que diz respeito aos fatores envolvidos na suspeição do processo saúde-doença ${ }^{21}$, além da inclusão da teoria do comportamento planejado ${ }^{15,16}$ e das características relacionadas com a amamentação, definidas pelas variáveis originariamente pesquisadas nos estudos que integraram a atual revisão sistemática.

Por sua vez, as variáveis relatadas nos estudos foram agrupadas em sete categorias: (a) características relacionadas à Teoria do Comportamento Planejado, (b) à gestação e amamentação (c) demográficas e socioeconômicas, (d) étnicas, (e) familiares, (f) de assistência à saúde, e (g) biológicas e hábitos (constituição física, doenças intercorrentes, estado fisiológico e nutricional).

\section{Resultados}

Isoladamente, os termos de buscas breastfeeding e aleitamento materno identificaram 24.985 artigos na PubMed e 3.284 na Lilacs. Os termos intention e intenção identificaram 5.726 (PubMed) e 1.106 artigos (Lilacs). Ao realizarmos a busca avançada com o auxílio de operadores boleanos combinando os termos breastfeeding e intention um total de 55 artigos foi identificado nas bases de dados; procedeu-se, em seguida, com a associação dos demais indicadores boleanos que provocaram exclusões de artigos de revisão (6), artigos que não avaliavam gestantes (26) e tese de doutorado (1). A etapa seguinte foi de leitura na integra dos 22 artigos, resultando na seleção de nove trabalhos que atendiam aos critérios de inclusão da atual revisão sistemática conforme demonstrado no diagrama de fluxo de seleção de estudos. Houve a perda de 1 artigo que não foi localizado para leitura (Figura 1).

As coletas de dados dos artigos selecionados ocorreram entre $1994^{3}$ e $2010^{22}$; um dos trabalhos não informou o ano da coleta ${ }^{23}$. Os desenhos dos estudos foram: intervenção randomizada e controlada (1), coorte (4), e corte transversal (4). Os locais de realização dos estudos foram: Estados Unidos (7), Reino Unido (1) e China (1). O Quadro 1 apresenta a descrição dos artigos selecionados de acordo com: autor, ano de publicação e país do estudo; tipo de estudo; amostra (número de gestantes); análise estatística efetuada e as variáveis associadas, ou não, à IMA. O resultado da qualificação metodológica dos 9 artigos que atenderam às condições de inclusão da atual revisão variou entre 12 e 21 pontos (Tabela 2).

Nos estudos selecionados foram identificados sessenta e oito variáveis cuja associação com a 


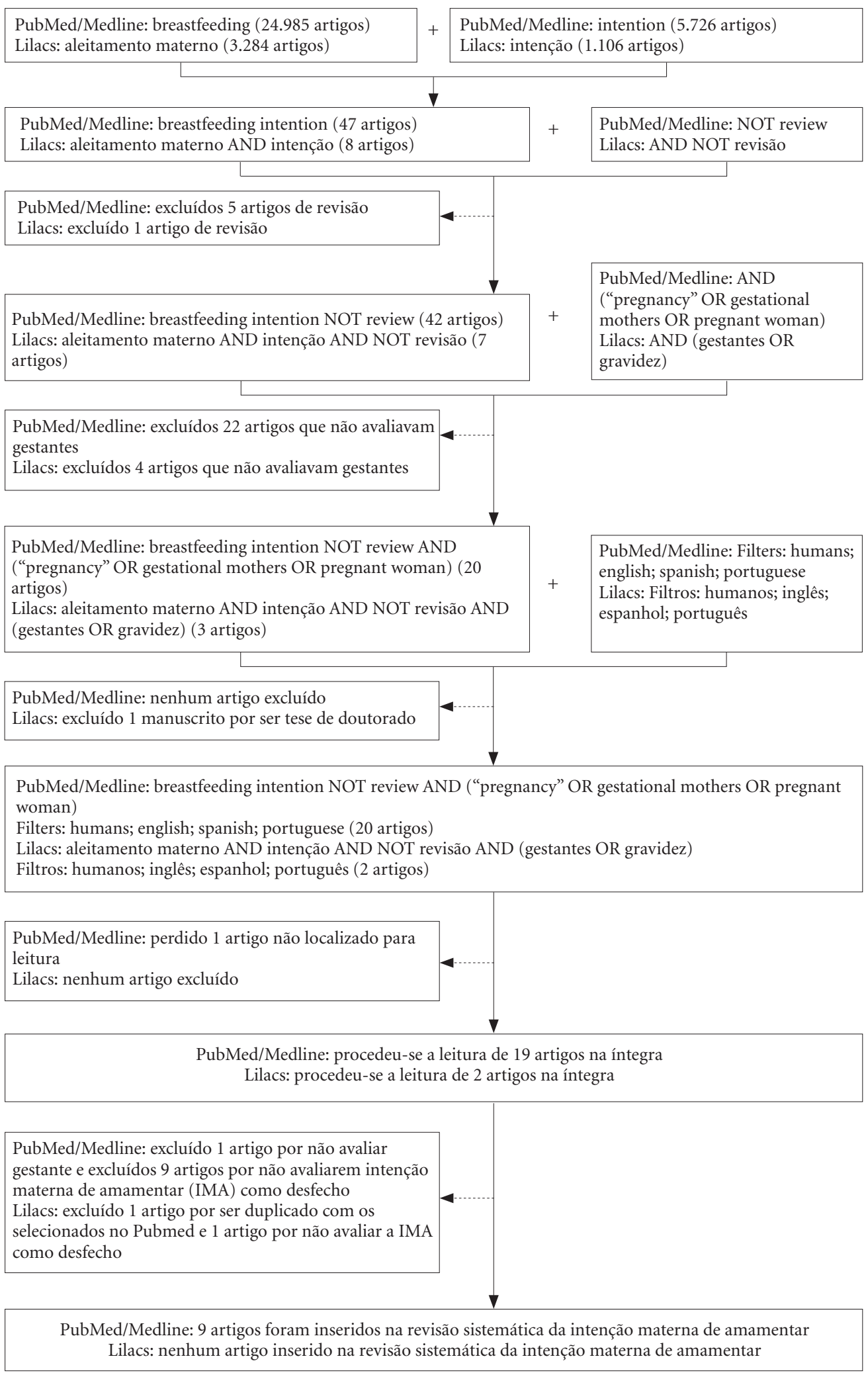

Figura 1. Diagrama de fluxo de seleção de estudos no Medical Literature Analysis and Retrieval System Online (PubMed/Medline) e na Literatura Latino-Americana e do Caribe em Ciências da Saúde (Lilacs). 
Quadro 1. Estudos incluídos na revisão sistemática dos fatores associados à intenção da gestante de amamentar.

\begin{tabular}{|c|c|c|c|c|c|}
\hline $\begin{array}{l}\text { Autor, ano de } \\
\text { publicação/ } \\
\text { País do } \\
\text { estudo }\end{array}$ & $\begin{array}{l}\text { Tipo de } \\
\text { estudo }\end{array}$ & $\begin{array}{c}\text { Amostra } \\
\text { (Número } \\
\text { de } \\
\text { gestantes) }\end{array}$ & $\begin{array}{c}\text { Análise } \\
\text { estatística }\end{array}$ & $\begin{array}{l}\text { Fatores estatisticamente } \\
\text { significantes }\end{array}$ & $\begin{array}{c}\text { Fatores sem significância } \\
\text { estatística }\end{array}$ \\
\hline $\begin{array}{l}\text { Stuebe and } \\
\text { Bonuk, } 2011 \\
\text { Estados } \\
\text { Unidos }\end{array}$ & $\begin{array}{l}\text { Intervenção } \\
\text { randomizado } \\
\text { e controlado }\end{array}$ & 833 & $\begin{array}{l}\text { Regressão } \\
\text { logística }\end{array}$ & $\begin{array}{l}\text { Não concordar com a suposição } \\
\text { de que "Fórmulas lácteas são tão } \\
\text { boas quanto leite humano". } \\
\text { Maior concordância com } \\
\text { a informação que crianças } \\
\text { amamentadas têm menos } \\
\text { probabilidade de desenvolver } \\
\text { infecções de ouvido, infecções } \\
\text { respiratórias, diarreia e obesidade. } \\
\text { Mulheres confortáveis em } \\
\text { amamentar em ambientes sociais. } \\
\text { Maior concordância com as } \\
\text { diretrizes de duração ótima do } \\
\text { aleitamento materno exclusivo. }\end{array}$ & $\begin{array}{l}\text { Idade materna. } \\
\text { Raça autorreferida / etnia. } \\
\text { Nacionalidade. } \\
\text { Participação materna em } \\
\text { programa de apoio à nutrição. } \\
\text { Escolaridade materna. } \\
\text { Paridade. } \\
\text { Duração da amamentação do } \\
\text { último filho. }\end{array}$ \\
\hline $\begin{array}{l}\text { Wambach, } \\
1997 \\
\text { Estados } \\
\text { Unidos }\end{array}$ & Coorte & 135 & $\begin{array}{c}\text { Análise de } \\
\text { caminho. } \\
\text { Regressão } \\
\text { logística }\end{array}$ & $\begin{array}{l}\text { Atitude positiva em relação à } \\
\text { amamentação durante o pré- } \\
\text { natal. } \\
\text { Controle comportamental } \\
\text { positivo quanto à amamentação, } \\
\text { percebido durante o pré-natal. }\end{array}$ & $\begin{array}{l}\text { Suporte social após o parto } \\
\text { (uso de escala com } 30 \text { itens). } \\
\text { Norma subjetiva em relação } \\
\text { à amamentação durante a } \\
\text { gestação. } \\
\text { Idade materna. } \\
\text { Renda familiar. } \\
\text { Escolaridade materna. } \\
\text { Experiência prévia com } \\
\text { amamentação. }\end{array}$ \\
\hline $\begin{array}{l}\text { Lee et al., } \\
2005 \\
\text { Estados } \\
\text { Unidos }\end{array}$ & $\begin{array}{l}\text { Corte } \\
\text { transversal }\end{array}$ & 2690 & $\begin{array}{l}\text { Regressão } \\
\text { logística }\end{array}$ & $\begin{array}{l}\text { Imigrante negra. } \\
\text { Mãe branca não latina. } \\
\text { Maior escolaridade materna. } \\
\text { Residir com o companheiro. } \\
\text { Primiparidade / primeira } \\
\text { gravidez. } \\
\text { Ausência de hábito de fumar. } \\
\text { Não residir em habitação pública. }\end{array}$ & $\begin{array}{l}\text { Idade materna. } \\
\text { Renda familiar. } \\
\text { Sintomas depressivos maternos. } \\
\text { Mãe com boa saúde física e } \\
\text { mental. } \\
\text { Qualidade da habitação. } \\
\text { Estabilidade da habitação. } \\
\text { Estado de privação (pobreza } \\
\text { extrema). } \\
\text { Estresse interpessoal (Escala de } \\
\text { medição de comportamento } \\
\text { violento do companheiro). } \\
\text { Estresse materno (uso de escala } \\
\text { com vários itens). } \\
\text { Apoio para execução de } \\
\text { atividades habituais. } \\
\text { Trabalho materno. }\end{array}$ \\
\hline
\end{tabular}

IMA foi investigada. Dentre essas, cerca da metade não esteve associada ao desfecho e $75 \%$ foi investigada em apenas um dos estudos, a exemplo das características relacionadas à Teoria do Comportamento Planejado. A variável idade materna foi relatada em todos os estudos (9), seguidas de 
Quadro 1. continuação

\begin{tabular}{|c|c|c|c|c|c|}
\hline $\begin{array}{c}\text { Autor, ano de } \\
\text { publicação/ } \\
\text { País do } \\
\text { estudo }\end{array}$ & $\begin{array}{l}\text { Tipo de } \\
\text { estudo }\end{array}$ & $\begin{array}{c}\text { Amostra } \\
\text { (Número } \\
\text { de } \\
\text { gestantes) }\end{array}$ & $\begin{array}{l}\text { Análise } \\
\text { estatística }\end{array}$ & $\begin{array}{l}\text { Fatores estatisticamente } \\
\text { significantes }\end{array}$ & $\begin{array}{c}\text { Fatores sem significância } \\
\text { estatística }\end{array}$ \\
\hline $\begin{array}{l}\text { Nommsen- } \\
\text { Rivers et al., } \\
2010 \\
\text { Estados } \\
\text { Unidos }\end{array}$ & Coorte & 532 & $\begin{array}{l}\text { Regressão } \\
\text { logística }\end{array}$ & $\begin{array}{l}\text { Autoconfiança na capacidade } \\
\text { de amamentar. } \\
\text { Conforto com a ideia de } \\
\text { amamentar o filho. } \\
\text { Desconforto com a ideia de } \\
\text { alimentação do filho com } \\
\text { fórmula. } \\
\text { Não ser afro-americana. }\end{array}$ & $\begin{array}{l}\text { Exposição à amamentação de } \\
\text { terceiros. } \\
\text { Participação em programa de } \\
\text { apoio à nutrição. } \\
\text { Idade materna. } \\
\text { Possuir seguro de saúde. } \\
\text { Escolaridade materna. }\end{array}$ \\
\hline $\begin{array}{l}\text { Humphreys } \\
\text { et al., } 1998 \\
\text { Estados } \\
\text { Unidos }\end{array}$ & $\begin{array}{l}\text { Corte } \\
\text { transversal }\end{array}$ & 1001 & $\begin{array}{l}\text { Regressão } \\
\text { logística }\end{array}$ & $\begin{array}{l}\text { Maior idade materna. } \\
\text { Maior escolaridade materna. } \\
\text { Ter experiência prévia com a } \\
\text { amamentação. } \\
\text { Etnia hispânica. } \\
\text { Receber orientações sobre } \\
\text { benefícios da amamentação } \\
\text { por membros da família e pai. } \\
\text { Receber orientações sobre } \\
\text { benefícios da amamentação } \\
\text { por consultores de lactação. } \\
\text { Cumplicidade com membros } \\
\text { da rede social que apoia a } \\
\text { amamentação. }\end{array}$ & $\begin{array}{l}\text { Estado civil. } \\
\text { Participação em programa de } \\
\text { apoio à nutrição. } \\
\text { Tempo de início do pré-natal. } \\
\text { Paridade. }\end{array}$ \\
\hline $\begin{array}{l}\text { Alexander et } \\
\text { al., } 2010 \\
\text { Estados } \\
\text { Unidos }\end{array}$ & $\begin{array}{l}\text { Corte } \\
\text { transversal }\end{array}$ & 176 & $\begin{array}{l}\text { Regressão } \\
\text { logística }\end{array}$ & $\begin{array}{l}\text { Primiparidade / primeira } \\
\text { gravidez. } \\
\text { Apoio e suporte do } \\
\text { companheiro. } \\
\text { Bom nível de conhecimento } \\
\text { materno sobre amamentação. }\end{array}$ & $\begin{array}{l}\text { Idade materna. } \\
\text { Escolaridade materna. } \\
\text { Raça / Etnia. } \\
\text { Estado civil. } \\
\text { Índice de massa corporal pré- } \\
\text { natal. } \\
\text { Tempo de início do pré-natal. } \\
\text { Decisão quanto à alimentação } \\
\text { do filho antes da gestação atual. } \\
\text { Época de aplicação do } \\
\text { questionário em relação ao } \\
\text { trimestre da gestação. } \\
\text { Planejamento de retornar ao } \\
\text { trabalho ou escola em dois } \\
\text { meses. } \\
\text { Conhecer pessoas que } \\
\text { amamentaram. } \\
\text { Mãe da criança ter sido } \\
\text { amamentada. } \\
\text { Conhecer os benefícios da } \\
\text { amamentação. } \\
\text { Considerar não haver problemas } \\
\text { em amamentar em público. } \\
\text { Ter boas expectativas quanto } \\
\text { à mudança corpórea com a } \\
\text { amamentação. }\end{array}$ \\
\hline
\end{tabular}




\begin{tabular}{|c|c|c|c|c|c|}
\hline \multicolumn{6}{|c|}{ Quadro 1. continuação } \\
\hline $\begin{array}{c}\text { Autor, ano de } \\
\text { publicação/ } \\
\text { País do } \\
\text { estudo }\end{array}$ & $\begin{array}{l}\text { Tipo de } \\
\text { estudo }\end{array}$ & $\begin{array}{c}\text { Amostra } \\
\text { (Número } \\
\text { de } \\
\text { gestantes) }\end{array}$ & $\begin{array}{l}\text { Análise } \\
\text { estatística }\end{array}$ & $\begin{array}{l}\text { Fatores estatisticamente } \\
\text { significantes }\end{array}$ & $\begin{array}{c}\text { Fatores sem significância } \\
\text { estatística }\end{array}$ \\
\hline $\begin{array}{l}\text { McLnnes et } \\
\text { al., } 2001 \\
\text { Reino Unido }\end{array}$ & Coorte & 995 & $\begin{array}{l}\text { Regressão } \\
\text { logística }\end{array}$ & $\begin{array}{l}\text { Experiência prévia com a } \\
\text { amamentação. } \\
\text { Residir com companheiro. } \\
\text { Não fazer uso de cigarro nos } \\
\text { últimos } 12 \text { meses. } \\
\text { Primiparidade / primeira } \\
\text { gravidez. } \\
\text { Maior idade materna. }\end{array}$ & $\begin{array}{l}\text { Estado de privação (pobreza } \\
\text { extrema). } \\
\text { Participação em programa } \\
\text { de apoio à nutrição com } \\
\text { recebimento de fórmulas lácteas. }\end{array}$ \\
\hline $\begin{array}{l}\text { Insaf et al., } \\
2011 \\
\text { Estados } \\
\text { Unidos }\end{array}$ & Coorte & 424 & $\begin{array}{l}\text { Regressão } \\
\text { de Poisson }\end{array}$ & $\begin{array}{l}\text { Ausência de estresse durante a } \\
\text { gestação. } \\
\text { Ausência de ansiedade durante } \\
\text { a gestação. } \\
\text { Ausência de sintomas } \\
\text { depressivos durante a gestação. } \\
\text { Maior escolaridade materna. } \\
\text { Menor número de filhos. } \\
\text { Ausência do hábito de fumar. } \\
\text { Trabalho materno durante a } \\
\text { gestação. }\end{array}$ & $\begin{array}{l}\text { Idade materna. } \\
\text { Renda familiar. } \\
\text { Possuir seguro de saúde. } \\
\text { Estado civil. } \\
\text { Residir com o companheiro. } \\
\text { Morar com outros adultos. } \\
\text { Uso de álcool. } \\
\text { Escala psicológica de } \\
\text { aculturação. } \\
\text { Idioma preferencial para falar e } \\
\text { ler (espanhol ou inglês). } \\
\text { Número de gerações após } \\
\text { imigração. }\end{array}$ \\
\hline $\begin{array}{l}\text { Lau, } 2009 \\
\text { China }\end{array}$ & $\begin{array}{l}\text { Corte } \\
\text { transversal }\end{array}$ & 2178 & $\begin{array}{l}\text { Regressão } \\
\text { logística }\end{array}$ & $\begin{array}{l}\text { Nascimento em Hong Kong. } \\
\text { Residência com tamanho } \\
\text { maior ou igual a } 30 \mathrm{~m}^{2} \text { (maior } \\
\text { área). } \\
\text { Início precoce do pré-natal. } \\
\text { Gravidez planejada. } \\
\text { Primiparidade / primeira } \\
\text { gravidez. } \\
\text { Casal com relacionamento com } \\
\text { menos conflitos. }\end{array}$ & $\begin{array}{l}\text { Morar com parentes. } \\
\text { Morar com avós. } \\
\text { Idade materna. } \\
\text { Escolaridade materna. } \\
\text { Tempo decorrido após a } \\
\text { imigração para Hong Kong. } \\
\text { Estado civil. } \\
\text { Trabalho materno fora do lar. } \\
\text { Renda familiar. } \\
\text { Número de filhos. }\end{array}$ \\
\hline
\end{tabular}

* O desfecho avaliado foi intenção da gestante de amamentar exclusivamente.

escolaridade materna (8), paridade (6), participação materna em programa de apoio à nutrição (4), estado civil (4) e renda (4). As características associadas à IMA em gestantes, relatadas em dois ou mais estudos, foram: primiparidade (4), maior nível de escolaridade materna (3), ausência do hábito de fumar (3), maior idade materna (2), experiência prévia com a amamentação (2) e residir com o companheiro (2). As variáveis que foram avaliadas em cada um dos nove estudos selecionados, associadas ou não com o desfecho, estão demonstradas na Tabela 3.

\section{Discussão}

A atual revisão sistemática da literatura, que sumarizou os fatores associados à IMA em gestantes, demonstrou que as características demográficas e socioeconômicas foram as mais estudadas 
Tabela 2. Qualificação dos nove estudos selecionados segundo os critérios e escores.

\begin{tabular}{|c|c|c|c|c|c|c|c|c|c|}
\hline Critérios de qualidade & 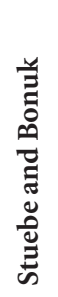 & 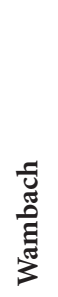 & 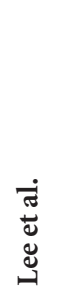 & 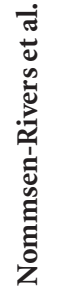 & 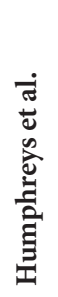 & 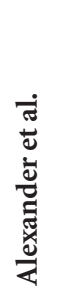 & 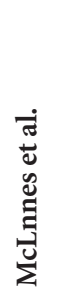 & 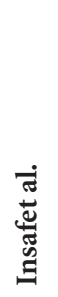 & ప్ \\
\hline Escala de validade interna (tipo de estudo) & 5 & 4 & 2 & 4 & 2 & 2 & 4 & 4 & 2 \\
\hline Resumo estruturado & 1 & 0 & 1 & 1 & 1 & 1 & 1 & 1 & 1 \\
\hline Introdução com embasamento e justificativa & 1 & 1 & 1 & 1 & 1 & 1 & 1 & 1 & 1 \\
\hline Método de recrutamento da população & 1 & 1 & 1 & 1 & 1 & 1 & 2 & 1 & 3 \\
\hline Seleção da amostra & 1 & 1 & 1 & 1 & 1 & 1 & 6 & 1 & 3 \\
\hline Instrumentos de coleta de dados & 3 & 3 & 0 & 3 & 0 & 1 & 0 & 3 & 3 \\
\hline Taxa de não-resposta informada & 1 & 1 & 1 & 1 & 1 & 1 & 1 & 1 & 1 \\
\hline Treinamento dos entrevistadores & 0 & 0 & 1 & 1 & 0 & 1 & 0 & 1 & 0 \\
\hline Método de medida de resultados definidos & 1 & 1 & 1 & 1 & 1 & 1 & 1 & 1 & 1 \\
\hline Realizado análise estatística & 1 & 1 & 1 & 1 & 1 & 1 & 1 & 1 & 1 \\
\hline Hipótese do estudo e viéses considerados & 1 & 1 & 1 & 1 & 1 & 1 & 1 & 1 & 1 \\
\hline Resultados interpretados segundo evidências & 1 & 1 & 1 & 1 & 1 & 1 & 1 & 1 & 1 \\
\hline Escala de generalização dos resultados & 1 & 1 & 1 & 1 & 1 & 1 & 1 & 1 & 3 \\
\hline Pontuação máxima & 18 & 16 & 13 & 18 & 12 & 14 & 20 & 18 & 21 \\
\hline
\end{tabular}

Adaptado de: Parry et al. ${ }^{19}$ e Taylor et al. ${ }^{20}$.

nos nove estudos selecionados. Apesar dessas variáveis não serem facilmente modificáveis por ações individuais, o seu conhecimento é fundamental na condução das medidas de intervenção direcionadas aos grupos populacionais vulneráveis, visando aumentar a prática do AM.

Dentre as características demográficas e socioeconômicas, a primiparidade ocupou posição de destaque, pois se associou positivamente à IMA em quatro ${ }^{3,7,9,10}$ dos seis estudos ${ }^{2,3,7,9,10,22}$. Esse achado pode refletir a disponibilidade materna de amamentar e cuidar de um único filho. Além disso, mulheres primíparas, certamente, não vivenciaram experiências prévias negativas com a amamentação, bem como não experimentaram o uso de fórmulas na alimentação dos filhos. De modo semelhante, o menor número de filhos foi preditor de IMA em um ${ }^{11}$ dos dois estudos ${ }^{10,11} \mathrm{em}$ que foi pesquisado.

A idade materna, característica que reflete experiências de vida e capacidade das mulheres em lidar com as mudanças e dificuldades inerentes ao período gestacional, à prática da amamenta- ção e aos cuidados com a criança, foi pesquisada em todos os estudos e se associou à IMA em apenas dois desses ${ }^{2,3}$, nos quais foi demonstrado que gestantes com maior idade manifestaram intenção de amamentar, quando comparadas às mães de menor idade.

A escolaridade materna, característica socioeconômica, esteve estatisticamente associada à IMA em três ${ }^{2,7,11}$ dos oito estudos que a avalia$\operatorname{ram}^{2,7,9-11,22,23,24}$. Um bom nível de instrução permite melhor assimilação materna das informações recebidas quanto às vantagens da amamentação ${ }^{25}$, com reflexos positivos nas prevalências do $\mathrm{AM}^{25}$.

Uma maior escolaridade materna, bem como a estabilidade econômica durante a gestação, são fundamentais para o planejamento de ações após o parto, a exemplo do tipo de alimentação. Embora não tenha sido demonstrada associação entre a renda e a IMA, nos estudos que a pesquisaram $^{7,10,11,23}$, mulheres que gozavam de melhores condições socioeconômicas durante a gestação, como residir em casas mais amplas ${ }^{10}$, não morar 
Tabela 3. Distribuição das variáveis associadas ou não com a intenção de gestantes de amamentar.

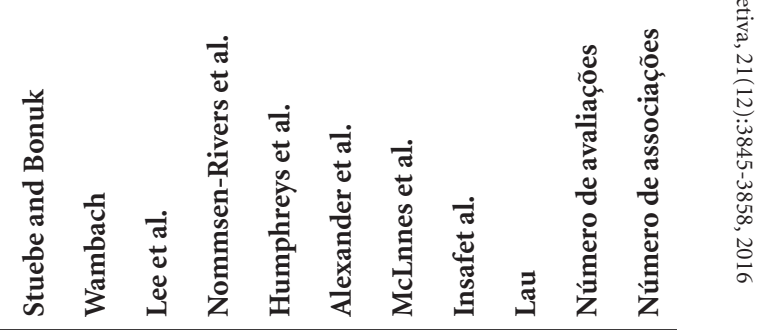

Características da teoria do comportamento planejado

Atitude

Atitude positiva em relação à amamentação no pré-natal.

Cumplicidade com membros da rede social que apoiam a

amamentação.

Mulheres confortáveis em amamentar em ambientes sociais.

Controle comportamental percebido

Controle comportamental positivo quanto à amamentação,

percebido durante o pré-natal.

Autoconfiança na capacidade de amamentar.

Conforto com a ideia de amamentar o filho.

Desconforto com a ideia de alimentar o filho com fórmula.

Bom nível de autoconhecimento sobre amamentação.

Norma subjetiva

Norma subjetiva em relação à amamentação na gestação.

Exposição à amamentação de terceiros.

Considerar não haver problemas em amamentar em público.

Características da gestação e da amamentação

Não concordar com a suposição de que "Fórmulas lácteas são tão

boas quanto leite humano".

Concordância em que crianças amamentadas têm menor chance de infecções e obesidade.

Maior concordância com as diretrizes de duração ótima do

aleitamento materno exclusivo.

Conhecer os benefícios da amamentação.

Duração da amamentação do último filho.

Ter experiência prévia com a amamentação.

Mãe da criança ter sido amamentada.

Conhecer pessoas que amamentaram.

Ter boas expectativas quanto à mudança corpórea com a

amamentação.

Decisão quanto à alimentação do filho antes da gestação atual.

Época de aplicação do questionário em relação ao trimestre da gestação.

Gravidez planejada.

Características demográficas e socioeconômicas

Maior idade materna.

Primiparidade / Primeira gravidez.

Menor número de filhos.

Participação materna em programa de apoio à nutrição.

Maior escolaridade materna.

Renda familiar.

Estado de privação (pobreza extrema).

Trabalho materno durante a gestação.

Planejamento de retornar ao trabalho ou escola em dois meses.

Residência com tamanho maior ou igual a $30 \mathrm{~m}^{2}$ (maior área).

Não residir em habitação pública.

S

S

S

S

$\mathrm{S}$

S

NS

S

S

S

NS

NS

列

NS

NS

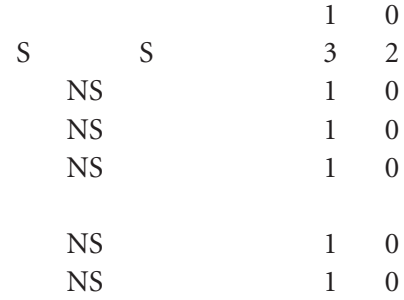

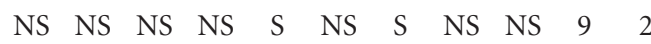

NS

NS

NS

Qualidade da habitação.

Estabilidade da habitação. 
Tabela 3. continuação

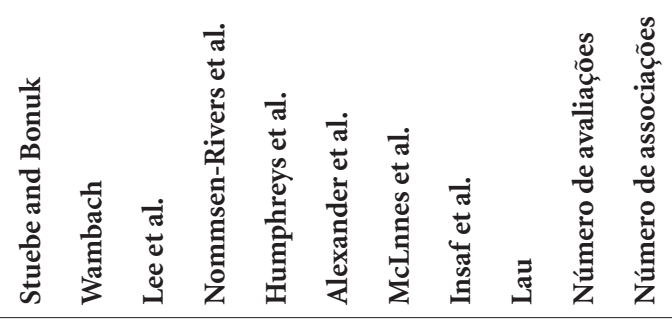

Características étnicas

Raça / Etnia.

NS

Etnia hispânica.

Ser de nacionalidade norte-americana.

NS

Mãe branca não latina.

Não ser afro-americana.

Nascimento em Hong Kong.

Tempo decorrido após a imigração para Hong Kong.

Escala psicológica de aculturação.

Idioma preferencial para falar e ler (espanhol ou inglês).

Número de gerações após imigração.

Características familiares

Suporte social após o parto (uso de escala com 30 itens).

Estresse interpessoal (escala de medição de comportamento violento do companheiro).

Apoio para execução de atividades habituais.

Apoio e suporte do companheiro.

Receber orientações dos benefícios da amamentação por familiares

ou pai.

Casal com relacionamento com menos conflitos.

Estado civil.

Residir com companheiro.

Morar com parentes.

Morar com avós.

Morar com outros adultos.

Características de assistência à saúde

Possuir seguro de saúde.

Receber orientações dos benefícios da amamentação por consultores.

Início precoce do pré-natal.

Características biológicas e hábitos

Índice de massa corporal pré-natal.

Ausência de estresse durante a gestação.

Ausência de sintomas depressivos durante a gestação.

Ausência de ansiedade durante a gestação.

Mãe com boa saúde física e mental.

Ausência do hábito de fumar.

Uso de álcool.

S

NS

$\begin{array}{lccc} & & 2 & 0 \\ & & 1 & 1 \\ & & 1 & 0 \\ & & 1 & 1 \\ & & 1 & 1 \\ & & 1 & 1 \\ & \text { S } & 1 & 1 \\ & \text { NS } & 1 & 0 \\ \text { NS } & & 1 & 0 \\ \text { NS } & & 1 & 0 \\ \text { NS } & & 1 & 0\end{array}$

NS

NS

$\begin{array}{ll}1 & 0 \\ 1 & 0\end{array}$

NS

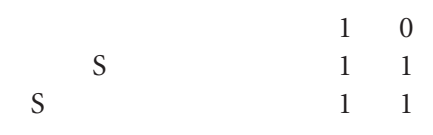

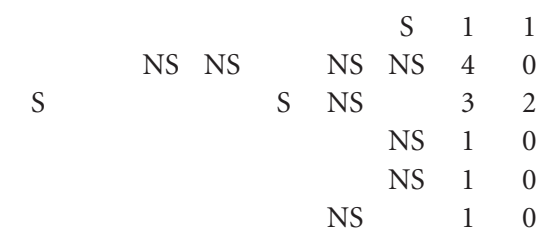

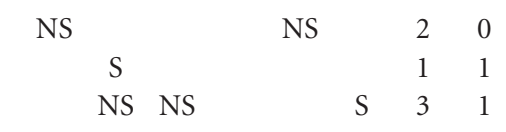

\begin{tabular}{|c|c|c|c|}
\hline \multicolumn{2}{|c|}{ NS } & & 1 \\
\hline NS & & $S$ & 2 \\
\hline \multirow[t]{2}{*}{ NS } & & $S$ & 2 \\
\hline & & $S$ & 1 \\
\hline NS & & & 1 \\
\hline \multirow[t]{2}{*}{$\mathrm{S}$} & $S$ & $\mathrm{~S}$ & 3 \\
\hline & & NS & 1 \\
\hline
\end{tabular}

$\mathrm{S}=$ significante; $\mathrm{NS}=$ não significante.

em habitação pública ${ }^{7}$ e que trabalhavam durante a gestação ${ }^{11}$ estiveram associados positivamente com o desfecho. Por sua vez, gestantes que manifestam preferência por alimentar os seus filhos com fórmulas, sobretudo aquelas de baixa renda, precisam de aconselhamento, ainda no pré-na$\mathrm{tal}^{26}$, quanto às vantagens e benefícios do $\mathrm{AM}^{27}$.

Outro fator que pode interferir na IMA são as características étnicas. Os estudos que avaliaram esta associação utilizaram diferentes abordagens, 
fato que dificultou a tabulação dos resultados na atual revisão sistemática, a exemplo do estudo conduzido na Califórnia, EUA, que avaliou amostra de raças/etnias e nacionalidade autorreferidas, distribuindo-as em cinco categorias: mulheres afro-americanas, asiáticas, hispânicas, brancas não hispânicas e mulheres com etnia mista (aquelas que autoidentificaram-se em mais de um grande grupo étnico). Nesse estudo foi informado que a IMA associou-se positivamente com gestantes de etnia não afro-americanas ${ }^{24}$.

Nascer fora dos EUA pode ser um fator de proteção para a definição da $\mathrm{IMA}^{28}$. Neste aspecto, para melhorar as taxas de AM, as mulheres americanas carecem de maior apoio à amamentação, não só dos familiares, como dos profissionais de saúde ${ }^{28}$. Entretanto, muitos desses estudos não esclarecem se o fato de amamentar está ligado ao grupo étnico ou se está sendo intermediado por fatores socioeconômicos. Em muitos países, a exemplo do Brasil, a população negra faz parte, de maneira geral, dos estratos de renda mais baixos da população, podendo as diferenças étnicas traduzir aspectos socioeconômicos desfavoráveis.

Características biológicas e hábitos de vida são também variáveis importantes na construção da IMA. Medir esses componentes é sempre um desafio para a pesquisa clínica, sobretudo quanto aos aspectos psicológicos que possuem medição específica e interagem com os desfechos através de uma complexa rede de fatores. Estresse, ansiedade e sintomas depressivos ${ }^{11}$ vividos por uma gestante, por exemplo, podem afetar os planos de amamentar, e associar-se ao fracasso do AM por dificultarem o início do processo de amamentação ${ }^{11}$. Diante disso, é preciso dar atenção especial às mães no período pós-parto, visto que esta é uma fase de risco para a manifestação de fatores psicológicos que podem afetar a IMA e o início do AM.

Dentre os hábitos de vida, a ausência do hábito de fumar foi um relevante fator associado positivamente à IMA ${ }^{3,7,11}$. É possível que mulheres fumantes decidam por não amamentar os seus filhos por saberem dos malefícios da nicotina para a saúde materna e fetal, e para não exporem o recém-nascido aos efeitos da passagem dessa substância por meio do leite materno ${ }^{29}$.

Quanto à "Teoria do Comportamento Planejado" foi identificada a influência positiva dos fatores relacionados à atitude $\mathrm{e}^{2,22,23}$, medidos por meio da cumplicidade de gestantes com membros da rede social ${ }^{2}$, e do sentimento de conforto de amamentar em ambientes sociais ${ }^{22}$; assim como das variáveis do controle comportamental percebido ${ }^{9,23,24}$, avaliadas por meio do nível do conhecimento materno sobre a amamentação ${ }^{9}$, da auto confiança da gestante na capacidade de amamentar, boa aceitação da ideia de amamentar e desconforto quanto ao uso de fórmulas de leite de vaca na alimentação do filho ${ }^{24}$. Porém, quando avaliada a norma subjetiva em relação à amamentação, não houve demonstração de associação ${ }^{23}$. Postula-se que a influência de cada um dos pilares da teoria sobre as intenções comportamentais varia em intensidade conforme o comportamento em foco, podendo interagir com os aspectos relacionados à gestação, amamentação e familiares. Entender as características modificáveis da Teoria Comportamental pode ajudar os profissionais de saúde na condução de ações de apoio ao $\mathrm{AM}^{30}$.

Entre as características relacionadas à gestação e amamentação, nos estudos revisados, associaram-se significativamente à IMA aquelas mães que concordam com as recomendações de duração ótima do aleitamento materno exclusivo $^{22}$, que reconhecem a superioridade do leite humano quando comparado ao artificial $^{22}$ e que tiveram experiência prévia positiva com o $\mathrm{AM}^{2,3}$.

Do mesmo modo, ter planejado a gravidez vigente foi um preditor de $\mathrm{IMA}^{10}$. O planejamento da gravidez permite às mulheres e aos homens escolherem quando querem ter o próximo filho, o número deles, o espaçamento entre eles, bem como discutir sobre a educação, a alimentação e a qualidade de vida. Nesse planejamento, o AM ocupa posição de destaque, pois, devido à sua excelência, protege a criança contra doenças a curto e a longo prazo e implica em menores gastos. Para aumentar a chance de IMA é preciso a organização dos serviços de saúde e a garantia de acesso das mulheres às unidades de planejamento familiar.

Quanto às características familiares, no que diz respeito ao relacionamento do casal, diferentes demandas psicológicas e emocionais são geradas para ambos os parceiros ${ }^{10}$, independente de estarem casados ou não.

Admite-se que as relações dos casais podem interferir na IMA por meio da atuação em um dos pilares da teoria do comportamento planejado, normas subjetivas maternas, ou ainda sob uma perspectiva direta, quando a mãe simplesmente atende à vontade paterna.

Ao avaliar 115 mães nas primeiras 24 horas após o parto, a aprovação da amamentação pelo pai associou-se com incidência de AM de 98,1\%, contra apenas $26,9 \%$, quando o parceiro era indiferente à escolha do tipo de alimentação do 
próximo filho ${ }^{31}$. Residir com o companheiro é um aspecto que facilita a participação do pai e o apoio dos familiares e contribui positivamente para a IMA ${ }^{2,7}$. Atualmente, o pai e, na sequência, a avó são as principais referências de apoio à mulher que amamenta ${ }^{2}$. Por outro lado, foi confirmada a associação entre violência do parceiro (sexual, física, ou psicológica) e menores chances de IMA entre mulheres joven ${ }^{29}$.

Características de assistência à saúde são importantes para a definição da IMA e para a prática do AM, sendo necessário o seu fortalecimento, tanto no período pré, quanto no pós-natal. O suporte de profissionais de saúde mediante o apoio emocional e orientações do ponto de vista prático, a exemplo de demonstrar à mãe como amamentar (a posição de colocar a criança no peito e a pega correta do complexo mamilo-areolar), e ensinar a técnica de ordenha do leite humano ${ }^{32}$ são fundamentais para a construção da IMA.

Lau et al. ${ }^{10}$ demonstraram associação positiva entre início precoce do pré-natal e IMA. Incentivar e ensinar o manejo da amamentação no pré-natal é importante para que as mulheres desenvolvam a autoconfiança em sua capacidade de amamentar, aprendam como superar obstáculos e experimentem êxitos com a amamentação no pós-parto imediato. Mulheres que receberam aconselhamento em amamentação tiveram taxas significativamente mais altas de IMA (61,1\%), em comparação com aquelas que não receberam $(34,7 \%, \mathrm{p}<0,005)^{33}$.

Refletir sobre a qualidade do suporte que as mães estão recebendo, não só dos familiares como dos profissionais de saúde durante o prénatal, é um desafio, visto que um dos estudos, nesta revisão, não demonstrou a associação positiva entre esses eventos e a IMA². Ademais, é preciso que as ações de promoção, incentivo e apoio à amamentação sejam repetidas em diferentes momentos, como no pré-natal, na sala de parto e após a alta hospitalar, para que sejam eficazes ${ }^{34}$.

\section{Considerações metodológicas}

Além das limitações metodológicas inerentes aos próprios modelos de estudos observacionais, que não permitem estabelecer uma relação de causalidade entre os fatores de exposição e o desfecho, é preciso considerar a possibilidade de viés de seleção induzido pelas ferramentas de busca, a exemplo de pesquisas que não estavam indexadas nas bases de dados pelas palavras convencionadas nos critérios de busca, como: desejo de amamentar, vontade de amamentar, querer amamentar, expectativa de amamentar; essa característica pode ter contribuído para a ausência de alguns estudos publicados, sobretudo os brasileiros, na atual revisão sistemática. Outro aspecto é o caráter do objeto pesquisado, ou seja, intenção de amamentar que, devido às influências, pressões e expectativas sociais e culturais, pode ter induzido respostas afirmativas, com superestimação dos resultados.

Ponto positivo da atual revisão foi realizar qualificação dos artigos, segundo critérios metodológicos que denotam a validade interna e externa de uma pesquisa; além de incluir estudos de diferentes países com distintas abordagens metodológicas, características que permitiram visualizar a complexa rede de fatores associados à IMA. Por outro lado, a heterogeneidade geográfica dos estudos incluídos na revisão pode ser uma limitação para a aplicabilidade dos resultados da atual revisão, pois os fatores de risco ou de proteção da IMA podem variar conforme as características individuais e ambientais e não se aplicar de forma apropriada em diferentes grupos populacionais.

\section{Conclusões}

A IMA é um comportamento construído ao longo da vida e antecede a prática da amamentação. São múltiplos os fatores associados e este evento e incluem as variáveis étnicas, socioeconômicas, demográficas e familiares, além dos hábitos de vida, das características biológicas, das relacionadas à gestação, amamentação e de assistência à saúde.

Reconhecer a importância do aleitamento materno e ter uma atitude positiva em relação à amamentação podem representar um passo fundamental na definição da IMA. Os profissionais de saúde são importantes figuras na definição dessa conduta; entretanto, medidas de intervenção devem ser iniciadas desde o pré-natal e estar voltadas, sobretudo, para as mulheres que não tenham planejado a gravidez, sem experiência prévia com amamentação, primíparas, mais jovens, de menor escolaridade, de menor estabilidade econômica, que não residam com o companheiro, que tenham conflito no relacionamento, que sejam fumantes, ou que vivenciem situações de ansiedade, estresse ou depressão.

Ausência de identificação de estudos, em diferentes países, que atendessem aos critérios de busca e de inclusão desta revisão, e o fato de nenhum dos artigos selecionados ter preenchido todos os critérios pré-estabelecidos de validade interna e externa, mesmo considerando a simplificada classificação utilizada, sinalizam para a necessidade de novas pesquisas. 


\section{Colaboradores}

TO Vieira, GO Vieira, CC Martins e LR Silva contribuiram com a concepção e o desenvolvimento do estudo, a coleta, a tabulação e a interpretação dos dados, a redação, a revisão crítica e a aprovação final da versão para publicação. GS Santana contribuiu na interpretação dos dados, redação, revisão crítica e aprovação final da versão para publicação.

\section{Referências}

1. Donath SM, Amir LH. Relationship between prenatal infant feeding intention and initiation and duration of breastfeeding: a cohort study. Acta Paediatr 2003; 92(3):352-356

2. Humphreys AS, Thompson NJ, Miner KR. Intention to breastfeed in low-income pregnant women: the role of social support and previous experience. Birth 1998; 25(3):169-174.

3. Mclnnes R, Love J, Stone D. Independent predictors of breastfeeding intention in a disadvantaged population of pregnant women. BMC Public Health 2001; 1(1):1013.

4. Mitra AK, Khoury AJ, Hinton AW, Carothers C. Predictors of breastfeeding intention among low-income women. Matern Child Health J 2004; 8(2):65-70.

5. Bonuck KA. Country of origin and race/ethnicity: impact on breastfeeding intentions. J Hum Lact 2005; 21(3):320-326

6. Al-Akour NA, Khassawneh MY, Khader YS, Ababneh AA, Haddad AM. Factors affecting intention to breastfeed among Syrian and Jordanian mothers: a comparative cross-sectional study. Int Breastfeed J 2010; 5(1):613.

7. Lee HJ, Rubio MR, Elo IT, McCollum KF, Chung EK, Culhane JF. Factors associated with intention to breastfeed among low-income, inner-city pregnant women. Matern Child Health J 2005; 9(3):253-261.

8. Leung TF, Tam WH, Hung ECW, Fok TF, Wong GWK. Sociodemographic and atopic factors affecting breastfeeding intention in Chinese mothers. J Paediatr Child Health 2003; 39(6):460-464.

9. Alexander A, O’Riordan MA, Furman L. Do breastfeeding intentions of pregnant inner-city teens and adult women differ? Breastfeed Med 2010; 5(6):289-296.

10. Lau Y. Breastfeeding intention among pregnant Hong Kong chinese women. Matern Child Health J 2009; 14(5):790-798.

11. Insaf TZ, Fortner RT, Pekow P, Dole N, Markenson G, Chasan-Taber L. Prenatal stress, anxiety, and depressive symptoms as predictors of intention to breastfeed among hispanic women. J Womens Health 2011; 20(8):1183-1192.

12. Marchand L, Morrow MH. Infant feeding practices: understanding the decision-making process. Fam Med 1994; 26(5):319-324.

13. Piazzalunga CRC, Lamounier JA. A paternidade e sua influência no aleitamento materno. Pediatr (São Paulo) 2009; 31(1):49-57.

14. Silva BT, Santiago LB, Lamonier JA. Apoio paterno ao aleitamento materno: uma revisão integrativa. Rev Paul Pediatr 2012; 30(1):122-130.

15. Ajzen I. Attitudes, personality, and behavior. $2^{\text {nd }}$ ed. Milton-Keynes: McGraw-Hill International; 2005.

16. Ajzen I, Madden TJ. Prediction of goal-directed behavior: attitudes, intentions, and perceived behavioral control. J Exp Soc Psycho. 1986; 22(5):453-474.

17. NCBI (National Center for Biotechnology Information) [Internet]. [acessado 2014 set 24]. Disponível em: http://www.ncbi.nlm.nih.gov/pubmed.

18. Biblioteca Virtual em Saúde (BVS) [Internet]. [acessado 2014 set 25]. Disponível em: http://regional.bvsalud.org. 
19. Parry LL, Netuveli G, Parry J, Saxena S. A systematic review of parental perception of overweight status in children. J Ambul Care Manage 2008; 31(3):253-268.

20. Taylor BJ, Dempster M, Donnelly M. Grading Gems: appraising the quality of research for social work and social care. Br J Soc Work 2007; 37(2):335-354.

21. Neto ASL, Cavalcanti LPG, Araújo WN, Rouquayrol MZ. Epidemiologia descritiva: características e possibilidades de uso. In: Rouquayrol MZ, Gurgel M, organizadores. Epidemiologia e Saúde. Rio de Janeiro: Medbook; 2013. p. 65-96.

22. Stuebe AM, Bonuck K. What predicts intent to breastfeed exclusively? Breastfeeding knowledge, attitudes, and beliefs in a diverse urban population. Breastfeed Med 2011; 6(6):413-420.

23. Wambach KA. Breastfeeding intention and outcome: a test of the theory of planned behavior. Res Nurs Health 1997; 20(1):51-59.

24. Nommsen-Rivers LA, Chantry CJ, Cohen RJ, Dewey KG. Comfort with the idea of formula feeding helps explain ethnic disparity in breastfeeding intentions among expectant first-time mothers. Breastfeed Med 2010; 5(1):25-33.

25. Boccolini CS. Determinantes sociais do aleitamento materno [tese]. Rio de Janeiro: Fundação Oswaldo Cruz; 2012.

26. Gurka KK, Hornsby PP, Drake E, Mulvihill EM, Kinsey EN, Yitayew MS, Lauer C, Corriveau S, Coleman V, Gulati G, Kellams AL. Exploring intended infant feeding decisions among low-income women. Breastfeed Med 2014; 9(8):377-384.

27. Newby R, Brodribb W, Ware RS, Davies PS. Infant feeding knowledge, attitudes, and beliefs predict antenatal intention among first-time mothers in Queensland. Breastfeed Med 2014; 9(5):266-272.
28. Persad MD, Mensinger JL. Maternal breastfeeding attitudes: association with breastfeeding intent and socio-demographics among urban primiparas. J Community Health 2008; 33(2):53-60.

29. Sipsma HL, Divney AA, Magriples U, Hansen N, Gordon D, Kershaw T. Breastfeeding intentions among pregnant adolescents and young adults and their partners. Breastfeed Med 2013; 8(4):374-380.

30. Cabieses B1, Waiblinger D, Santorelli G, McEachan RR. What factors explain pregnant women's feeding intentions in Bradford, England: a multi-methods, multi-ethnic study. BMC Pregnancy Childbirth 2014; 14:50.

31. Littman H, Medendorp SV, Goldfarb J. The decision to breastfeed. The importance of father's approval. Clin Pediatr (Phila) 1994; 33(4):214-219.

32. Kervin BE, Kemp L, Pulver LJ. Types and timing of breastfeeding support and its impact on mothers' behaviours: types and timing of breastfeeding support. $J$ Paediatr Child Health 2010; 46(3):85-91.

33. Sable M. Prenatal lactation advice and intention to breastfeed: selected maternal characteristics. J Hum Lact 1998; 14(1):35-40.

34. Oliveira BC, Rodrigues DA, Lamounier JA. Intenção de amamentar e a prática de amamentação em maternidades de Belo Horizonte. Rev Med Minas Gerais 2005; 15(4):225-228.

Artigo apresentado em 19/05/2015

Aprovado em 25/09/2015

Versão final apresentada em 27/09/2015 